\title{
Best Practices for Reliable and Robust Spacecraft Structures
}

\author{
I. S. Raju ${ }^{1}$, P. L. N. Murthy ${ }^{2}$, N. R. Patel $^{3}$, P. J. Bonacuse ${ }^{4}$, K. B. Elliott' ${ }^{5}$ S. A. Gordon ${ }^{6}$, \\ J. P. Gyekenyesi ${ }^{7}$, E. O. Daso ${ }^{8}$, P. Aggarwal ${ }^{8}$, and R. F. Tillman ${ }^{9}$ \\ NASA Engineering and Safety Center \\ NASA Langley Research Center \\ Hampton, Virginia 23681
}

\begin{abstract}
A study was undertaken to capture the best practices for the development of reliable and robust spacecraft structures for NASA's next generation cargo and crewed launch vehicles. In this study, the NASA heritage programs such as Mercury, Gemini, Apollo, and the Space Shuttle program were examined. A series of lessons learned during the NASA and DoD heritage programs are captured. The processes that "make the right structural system" are examined along with the processes to "make the structural system right". The impact of technology advancements in materials and analysis and testing methods on reliability and robustness of spacecraft structures is studied. The best practices and lessons learned are extracted from these studies. Since the first human space flight, the best practices for reliable and robust spacecraft structures appear to be well established, understood, and articulated by each generation of designers and engineers. However, these best practices apparently have not always been followed. When the best practices are ignored or short cuts are taken, risks accumulate, and reliability suffers. Thus program managers need to be vigilant of circumstances and situations that tend to violate best practices. Adherence to the best practices may help develop spacecraft systems with high reliability and robustness against certain anomalies and unforeseen events.
\end{abstract}

\section{Introduction}

NASA is currently in the process of developing the next generation crewed and cargo launch vehicles and spacecraft to return to the moon and beyond. With the experience and knowledge base available from past similar programs, a document that captures salient aspects of successful programs is being developed. This document serves as an important guide in evaluating next generation and future spacecraft concepts and proposals. As a part of this guide, guides for individual technical disciplines are being developed. Reliable and robust structural systems design is one of these technical disciplines. The structures document describes pertinent issues, best practices, errors, miss-steps, lessons learned, and summarizes the previously used design processes (tools and standards) for the structures discipline.

\footnotetext{
${ }^{1}$ Langley Research Center, Hampton, Virginia, Fellow AIAA

${ }^{2}$ Glenn Research Center, Cleveland, Ohio, Associate Fellow AIAA

${ }^{3}$ The Aerospace Corporation, El Segundo, California, Senior Member AIAA

${ }^{4}$ Army Research Laboratory at NASA Glenn Research Center, Cleveland, Ohio

${ }^{5}$ Langley Research Center, Hampton, Virginia

${ }^{6}$ Goddard Space Flight Center, Greenbelt, Maryland

${ }^{7}$ Glenn Research Center, Cleveland, Ohio, Member AIAA

${ }^{8}$ Marshall Space Fight Center, Huntsville, Alabama

${ }^{9}$ Jet Propulsion Laboratory, Pasadena, California
} 
Structural systems provide the basic framework to distribute external and internal loads resulting from all flight loads, ground loads, and associated operational and environmental loads. The primary objective of a structural system is to remain intact and experience minimal deformation when exposed to various environments, including ground processing, testing, launch, on-orbit, and re-entry operations. Structural systems also provide containment for pressures as in pressure vessels, pressure components, and pressurized structures. Structures tend to be a dependent subsystem in the sense that many requirements flow to structures from other subsystems. Space systems are very complex products of multiple disciplines and therefore are multidisciplinary, and therefore require a multidisciplinary analysis and optimization approach to capture various system interactions and sensitivities in order to obtain optimum system solutions, develop flight constraints, and validate and verify the system architecture. As a result, as illustrated in Figure 1, the development of the structural system is a complex iterative design process.

This paper outlines the best practices that are essential to the design and production of reliable and robust spacecraft structural systems. First, the NASA heritage programs are examined. Lessons learned from these heritage programs are captured. Next the processes that need to be used to "make the right structural system" are examined. Third, the processes that need to be followed to "make the structural system right" are addressed. In addition, a brief review of methods for assessing reliability and risk for structural systems is provided. Finally, the lessons learned and the best practices are presented.

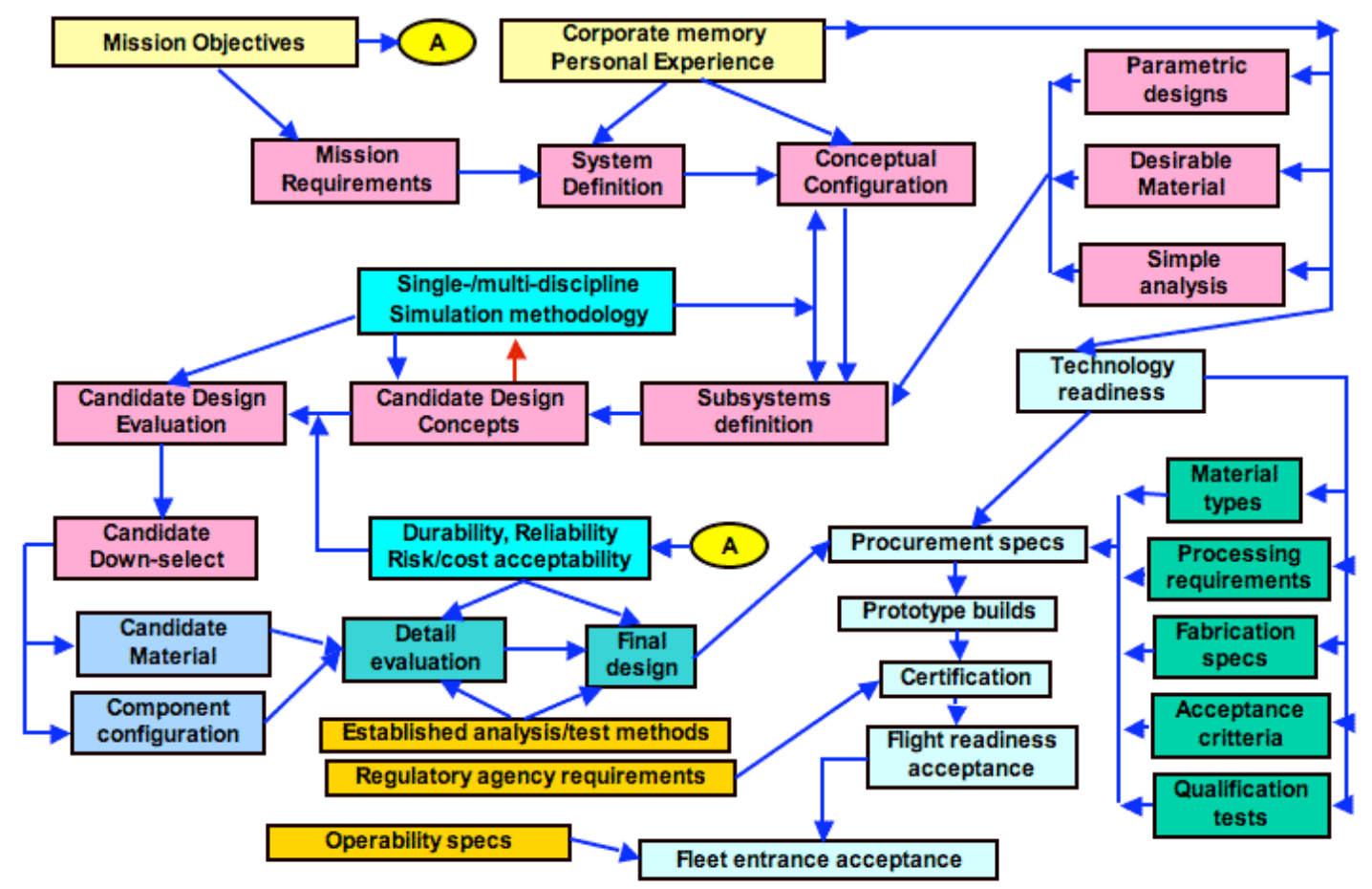

Figure 1. Complex interactions involved in structural system design. 


\section{Heritage Programs}

Heritage programs such as Mercury, Gemini, Apollo, and the Space Shuttle program (see Figure 2) are first examined [1-8]. A study of these programs resulted in several lessons learned. They can be briefly summarized as follows:

- Design deficiencies result from the inability to predict load paths and load distributions accurately. All load paths in a complex structure may be difficult to discern. Thus careful analysis followed by a rigorous test program should be conducted to uncover any design deficiencies.

- Testing of components requires care. Whenever possible, test hardware should be structurally similar to flight hardware. Special attention needs to be given to interfaces and boundaries to ensure that proper boundary conditions are imposed on the system or component.

- Despite advances in analysis techniques, modeling and simulation verification and validation is a vital part of insuring the reliability of structural systems.

- A building block approach is required to design and build reliable complex structures. Key steps that need be followed are:

- Fully characterize special materials used in the structures and structural components.

- Develop and validate, to the extent possible, accurate environmental predictions and verify the techniques used in the predictions.

- Develop accurate structural dynamic and stress models and validate their predictions. Avoid extrapolations of models and results.

- Develop a fracture control/nondestructive evaluation program.

- Develop extensive verification and validation procedures for:

- Modeling and analysis

- Coupon tests, subcomponent tests, component tests, full scale tests, and flight tests

- Analysis and test correlation

- Develop rigorous manufacturing and quality control procedures. 


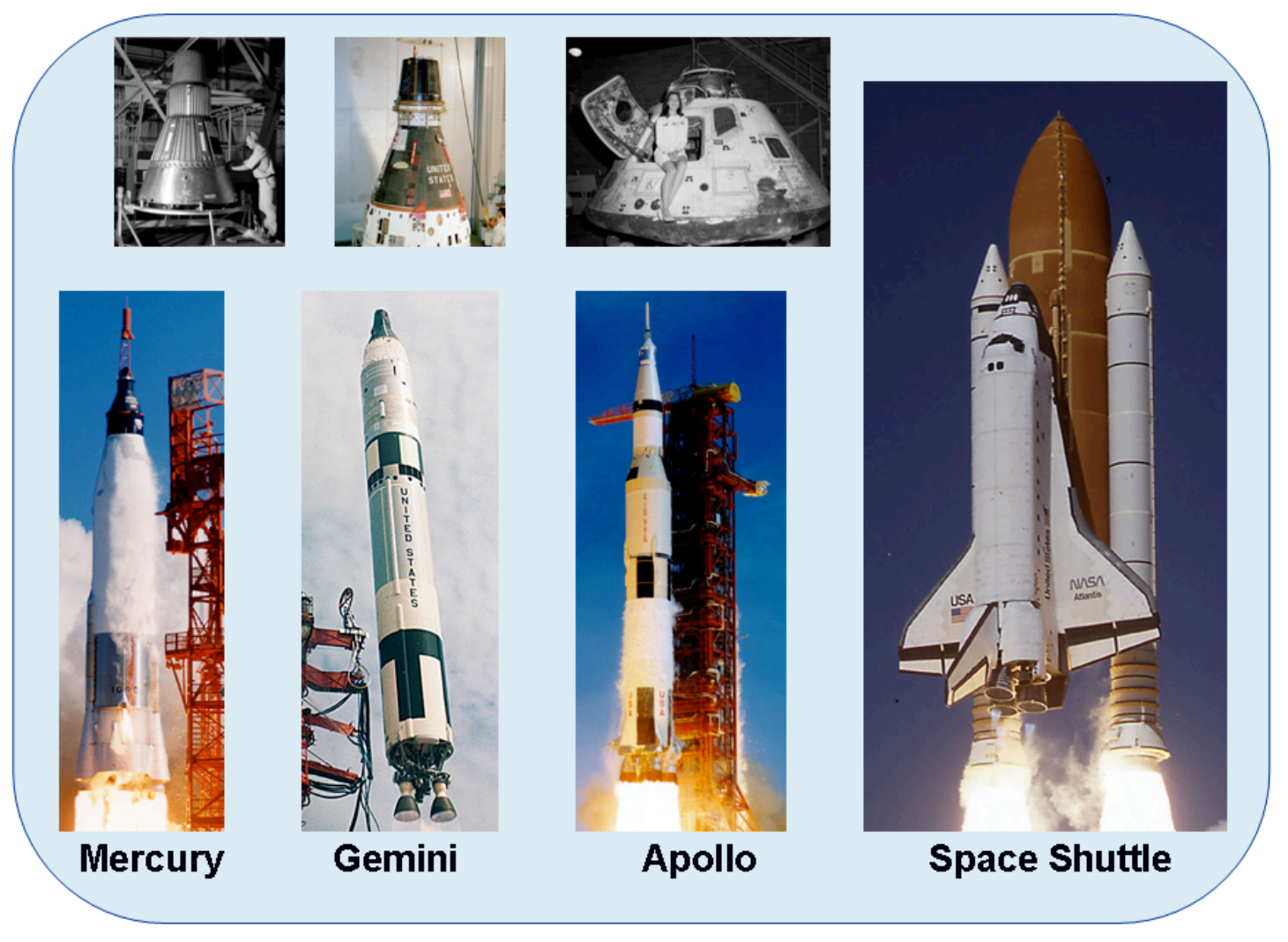

Figure 2. NASA Heritage Space Programs

\section{Making the Right Structural System}

Structural components (other than consumable or life limited items) are intended to have sufficient durability to perform adequately over the expected service life of the system. Structural systems deteriorate and/or develop damage due to a single cause or a combination of causes such as: (a) the design is inadequate for the applied loading and environment (conceptual design or calculation error), (b) the loading (amplitude, frequency, and/or interactions) is not well understood or underestimated, (c) the effects of environment are underestimated (requirements specification error), (d) a flaw in the materials or manufacturing is undetected (quality control and/or inspection error), and (e) unexpected damage occurrs through unforeseen means (e.g. handling damage). Thus to make the right structural system, several best practices, such as the establishment and understanding of the proper design and mission requirements, the implementation of trade studies, and the creation of sufficient verification and validation studies, need to be instituted.

\section{Design Requirements:}

The primary purpose of a structure is to protect spacecraft systems and to ensure that the system remains intact by maintaining the relative position of components under servicelife loads and environments. Thus the fundamental requirement of any space structure is 
to maintain structural integrity throughout the life of the structure. The process of defining structural requirements for new spacecraft typically begins with a review of previous development efforts and applicable technical standards. Both of these sources should be mined for appropriate design constraints, testing requirements, methodologies, and procedures. Care should be taken in selecting the requirements that will appear in the final system specification. All requirements should add value, be clear in their intention, and should not overly constrain the design and development. The list of requirements should be determined through an active negotiation process between the project management and the appropriate technical community. As a minimum, all NASA programs should evaluate NASA and other government standards for applicability. In general, these standards capture best practices, and they represent the starting point for the design, analysis, and verification of structural systems within NASA and other Government Agencies. If a program intends to deviate from the approach outlined in the NASA standards, then it will most likely require that documentation of the technical rationale or waiver be provided to the organizations performing technical oversight of the program during the formal review process.

\section{Mission Requirements:}

Performance: Structural design, including the implementation of new technologies, is driven by the system performance requirement goals. These performance requirements are driven by the mission requirements. Demanding performance requirements combined with volume and weight constraints often lead to greater sensitivities to design uncertainties. Design uncertainties exist in material properties, environments, loads, analyses, testing, and manufacturing. It is preferred to have a linear sensitivity of performance to these parameters. On the other hand, a high performance design may require nonlinear dependence on these parameters. In that case, great care must be taken to characterize material properties, define environments, and validate analyses. Manufacturing, quality control and assurance, and acceptance criteria must be enhanced to account for additional uncertainty. On the other hand, robust or conservative designs can be used at a price of higher weight and possibly lower performance. The optimum design choice probably lies between the two extremes. Trade and sensitivity studies must be performed to determine the trade offs and select the optimum design.

Environments: The structural system is designed and tested to withstand all pertinent environmental conditions, naturally occurring and induced, to which the system will be subjected during its life-cycle. These life-cycle environments should be identified as early as possible in the structural design process, and appropriate loading conditions should be defined as requirements for design and testing. Typical environments include production, testing, integration, storage, transportation, launch, ascent, thermal, radiation, meteoroid impact, vacuum, dust or contamination, re-entry, and landing. Care should be taken to consider load uncertainties, combine environmental effects, and contingency load cases. The structural system should also be designed to withstand the cumulative effects of the environments without loss of mission performance. 


\section{Trade Studies:}

The preliminary requirements for the design of a structural system typically involve the definition of mass allowables, volume constraints, and the specification of both static and dynamic design loads. These requirements stipulate the trade space for evaluating different structural concepts. In addition, manufacturability, inspectability, and cost (both initial and lifetime) may be additional constraints on the trade space. In most cases, the structural design trades are aimed at minimizing vehicle weight while showing positive margins under the specified design loads and providing sufficient stiffness to meet mission goals. One of the first trades in developing a preliminary structural design is to define the load paths and the type of structure that will sustain the design loads. Trade studies can also be performed to evaluate different material types (for example, composite vs. metal), the implementation of new technologies, and different construction methods.

Verification and Validation (V/V): Verification and validation are terms often used in relation to the qualification of reliable structures. The terms verification and validation are often misused or used interchangeably. NASA system engineers define verification as "proof of compliance with specification as determined through a combination of test, analysis, and demonstration" [9]. Validation is defined as "proof that a product accomplishes the intended purpose as determined through a combination of test, analysis, and demonstration" [9]. In other words; verification is demonstrating that the product meets the design requirements, and validation is demonstrating that the product meets the goals of the intended application. These definitions originate at the system level and primarily apply to hardware products. A second set of definitions are commonly used in reference to computational models. Model verification, as defined by AIAA, ASME, and DoD [10-12], is "the process of determining that a computational model accurately represents the underlying mathematical model and its solution." Model validation is "the process of determining the degree to which a model is an accurate representation of the real world from the perspective of the intended uses of the model." In this case, verification is ensuring the computational model is correct in terms of the governing equations (stress, strain, motion); validation is ensuring the modeling effort captures the physics of the intended application. Producing reliable structures requires meeting both sets of definitions. For example, computational models need to endure sufficient V/V to define or reduce uncertainty and demonstrate sufficient accuracy to support program decisions. This is particularly important when computational models are to be used for product $\mathrm{V} / \mathrm{V}$. Best practice dictates that all structural systems should undergo a rigorous $\mathrm{V} / \mathrm{V}$ process.

The following are examples of lessons learned taken from various past aerospace programs that relate to "making the right structural system":

- Document engineering requirements as clearly as possible. All requirements, including seemingly minor changes, should be clearly documented and tracked in order to avoid misinterpretation.

- In a totally new system, requirements may have to be continually reviewed for applicability. Requirements may change as a result of trade studies or design maturity. 
- Each requirement should be traceable to a compliance matrix. All test data should be inspected for trends and "out of family" values, even when all values are within the expected range. Anomalous data should be thoroughly investigated.

- Impact of requirements changes for a subsystem should be properly evaluated on the system and interfacing subsystems.

- Review out-of-flow processes to ensure no steps are bypassed.

- Spacecraft must be designed to withstand worst-case life-cycle environments. All possible load combinations should be considered. Credible mission failure scenarios should be considered in evaluating the failure modes of the structure.

\section{Making the Structural System Right}

The key aspects for reliable and robust structures are design, analysis, manufacturing and process control, testing, and quality assurance. Each topic must be properly addressed to "make the structural system right".

Design:

Primary and secondary structures of space systems are designed to provide sufficient strength, rigidity, and other characteristics required to sustain the critical loading conditions without damage or degradation of performance throughout their service life. Several key design aspects necessary to ensure a reliable structure are structural integrity requirements, fatigue and fracture control, factors of safety, material properties, and tolerance requirements.

\section{Analysis:}

Structural analyses are performed to predict structural response to the critical loads and environments anticipated during the service life of the structure. Typical analyses include investigations of fatigue, safe-life, and fail-safe considerations. These analyses are important to establish the service life, tolerance of the structure to defects, design margins, and residual strength. To ensure a reliable structure, it is important to verify the analysis models and validate the analysis predictions over the range of use.

\section{Manufacturing and Process Control:}

The design of reliable and robust structures requires well-characterized fabrication processes and procedures. The fabrication process for each structural item is a controlled, documented process. Proven processes and procedures for fabrication and repair are needed to preclude damage or material degradation during material processing and manufacturing operations. An inspection plan is required to identify all key process parameters essential for design verification. In-process inspection or process monitoring are needed to verify setup and acceptability of critical parameters during the manufacturing process. 


\section{Testing:}

Demonstrations are required to ensure that a structural system meet both mission and regulatory requirements. These demonstrations can be performed in four ways: 1) by heritage/similarity, 2) by analysis, 3) by qualification testing, and 4) by a combination of 1,2 , and 3. Qualification through heritage/similarity is not a reliable process without adequate analyses and tests to conclusively demonstrate similarity in materials, loads, environments, and responses. Qualification through analysis may be used when testing cannot demonstrate a target environment, such as zero-g or combined load effects, or the tests required are hazardous or unrealistic in terms of cost and schedule. By far the best approach to qualification is through testing. The mantra for a qualification-testing program should be "Test what you fly, and fly what you test."

\section{Quality Assurance:}

A quality assurance program based on a comprehensive study of the product and engineering requirements is established to ensure that necessary nondestructive inspection and acceptance proof tests are performed effectively. The program ensures that no damage or degradation occurred during material processing, fabrication, inspection, acceptance tests, shipping, storage, assembly, and operational use and refurbishment, and that defects that could cause failure are detected or evaluated and corrected. Acceptance proof tests are conducted on pressure vessels, pressurized structures, and composite structures for verification of workmanship.

\section{Reliability Assessment Methods}

Risk and reliability are complementary terms. Reliability is a quantified probabilistic assurance that a system or a product/device (or structure) will perform satisfactorily (i.e. will not fail, and will satisfy performance requirements) during its intended lifetime under specified operating conditions. Probability of failure refers to likelihood that the system will fail to satisfy the designated performance requirement. Risk, on the other hand, combines the probability of failure and the consequence of failure. Risk is generally defined as a product of the probability of failure and the cost (consequence) of failure.

A distinction also needs to be made between probability and statistics. Statistics is the mathematical quantification of uncertainty (mean, standard deviation, and other higher moments) mainly through the analysis of measured data. Probability theory uses statistical data to quantify the likelihood of occurrence of specific events.

Many structural systems (particularly space propulsion structures) are one-of-a-kind and hence have little or no learning curve. Generally, space propulsion systems are relatively large and expensive; and often they are not fully tested prior to their use nor are they tested repeatedly to create a statistical database. These propulsion systems may also have relatively long exposure periods and are designed for low risk. Often there is little or no redundancy is in these systems because of cost and weight considerations. These factors make the reliability analysis of these systems extremely challenging. 


\section{Margins and Factors of Safety:}

Engineers have always recognized the existence and presence of uncertainty in the analysis and design of structural systems. These uncertainties can arise due to human factors (error in analysis and/or fabrication), limitations in technology, inherent randomness in the material properties, the environment in which the system operates, and the specific utilization of the structural system. Traditionally, uncertainty has been accounted for by using safety factors and/or knock-down factors. Structural reliability and robustness is improved by increasing the safety margins for critical structures (with a cost and weight penalty) and by practicing rigorous quality assurance (QA) and quality control (QC) techniques.

Depending upon the nature of their criticality, space system structures and structural components are designed using either a fail-safe or a safe-life design philosophy. Failsafe systems, sometimes referred to as fool-proof systems, are designed such that their failure does not affect other components and systems. In the safe-life design philosophy, systems are designed to survive a specific design life with a chosen reserve.

Historically, the use of empirical safety factors has been the prevalent method of making designs more reliable. However, deterministic safety factors do not provide a quantitative measure of risk. In contrast, probabilistic analysis methods can provide this information.

Traditionally, safety factors are estimated based on rules-of-thumb and experience and are intended to be conservative. Selection of safety factors is insensitive to required reliability. It is possible to establish relationships between the traditional safety factors and the more rigorous probabilistic methods provided the underlining distributions and the statistical parameters for various design factors are known. Lately, there has been a push to relate safety factors to probabilistic or statistical methods. Safety factors that are based on standard probabilistic analyses provide a transparent approach to the end user recognizing the statistical nature of material properties and stress, the applicability of the failure theory, fidelity of the analysis techniques, and the required reliability. In applying this methodology, all design parameters of interest are typically assumed to have normal distributions [13]. This is an approximation, but in the absence of adequate data, the normal distribution assumption is advantageous in that it can be fully characterized by just two parameters: the mean and the variance. Since the applied stress and allowable strength are statistical in nature and assumed to be normal (with known parameters), the safety factor can be shown to be a function of probability of failure, mean and standard deviation of strength, and mean value and standard deviation of applied stress.

\section{Probabilistic Approaches:}

Usually, in risk-based design methodologies, a traditional load and resistance model is used. In its most fundamental form, design safety is ensured by requiring that the resistance is greater than the load. The safety factor is defined as the resistance divided by load. The determination of resistance and load distributions depend on the specific application. In aerospace applications, another measure of safety, margin of safety 
(MOS), is often used. Positive values of MOS indicate safe designs, and negative values indicate unsafe designs.

In a probabilistic analysis, the design safety is ensured by requiring that the overlap between the load and resistance (strength) probability distribution curves be minimized within the constraints of economy. Figure 3 illustrates and compares the traditional and probabilistic design methodologies. The probability of failure is defined as the total area of the overlap [14]. The shapes of the curves are represented by probability density functions. In certain situations, the resistance distribution curve needs to account for more than the traditional strengths. For example, when the structural components are subjected to variable amplitude, high frequency loading where fatigue is the primary failure mechanism, the resistance needs to properly account for history dependent fatigue damage accumulation.

\section{Traditional Factor Of Safety Approach}

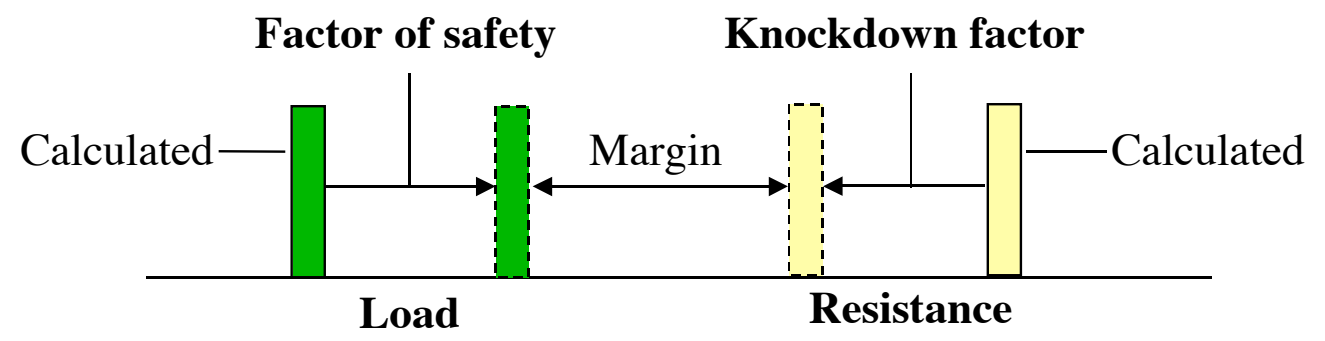

\section{Probabilistic Approach}

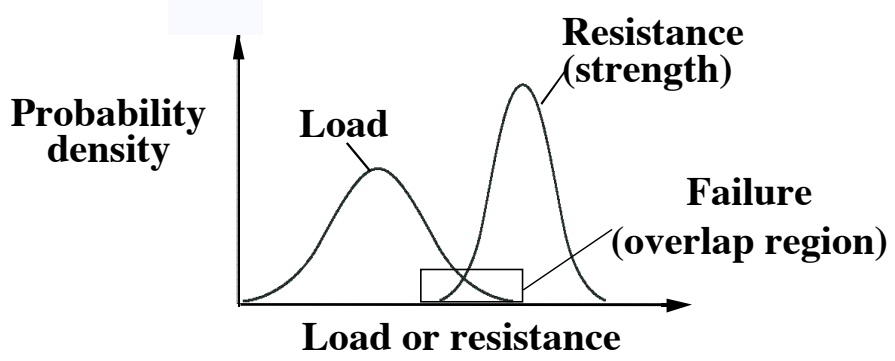

Figure 3. A comparison of traditional vs. probabilistic design methodologies

Generally, the methods to quantify the element level reliability (component reliability) can be broadly grouped into four categories as, (1) first-order reliability methods (FORM) and second-order reliability methods (SORM), (2) Monte Carlo simulation and its derivatives like efficient sampling methods etc., (3) response surface approaches, and (4) sensitivity-based probabilistic finite element analysis. References 14-17 provide details on these methods. 
The state-of-the-art in the area of structural reliability assessment has improved significantly in the past two decades both in component and system level reliability estimation. Many commercial finite element codes have adopted probabilistic analyses methods $[18,19]$. These methods have been applied successfully in the areas of material uncertainty characterization, probabilistic fracture mechanics, probabilistic fatigue analysis, and probabilistic analysis of structural systems. However, these methods are computationally intensive. The challenge that remains is to synthesize, adapt, and simplify research efforts into practical and efficient methods that can be used for a variety of engineering applications.

\section{Best Practices Based on Lessons Learned}

The following are examples of the best practices developed based on the lessons learned taken from various past aerospace programs. Adherence to these practices will help ensure the development of reliable and robust structural systems.

Qualification

- Thoroughly evaluate heritage systems and data (test and analysis) as well as the applicability of using "existing" or "flight proven" equipment.

- Unexpected hardware behavior in test and/or flight is often a sign of impending failure and must be thoroughly investigated. Perform thorough post-flight analyses.

- Replacement materials should be sufficiently tested under conditions that realistically simulate flight conditions, and the results should be correlated with those exhibited by the original material systems.

- Study past anomalies that involved similar designs or technologies and implement appropriate corrective actions.

- Safeguard flight hardware against inadvertent damage due to handling and over-testing.

- Do not succumb to launch schedule pressure and compromise engineering recommendations.

Analysis and Testing

- All design changes must be thoroughly analyzed and tested.

- Analysis should properly account for all flight environments.

- Inaccuracies in material properties, structural loads, and environments continue to threaten mission success. Validation of material properties, structural loads, and environments through rigorous test campaigns is the best method of insuring reliable structures.

- Test failures must be thoroughly investigated and the root causes of the test anomalies ascertained and understood.

- Verify field installation of all single point failure items. 
Design, Manufacturing, and Assembly

- Thoroughly verify the interfaces of all subcontracted items.

- Honeycomb structures should be vented wherever possible. If un-vented design cannot be avoided, sufficient testing including development, qualification, and proof tests should be conducted under applicable temperature and vacuum conditions.

- Changes and some non-conformances typically do not go through material review board processes. All changes and discrepancies should be properly evaluated.

- $11^{\text {th }}$-hour modifications at the launch site require thorough evaluations.

- Protect the flight hardware from handling and transportation damage. Provide ample checks for damage detection.

- Design hardware to minimize the areas that cannot be inspected, and avoid the use of potential contaminants whenever possible. Account for all loose materials used during assembly.

\section{Concluding Remarks}

Spacecraft structural systems are complex and have multiple interacting components. As such these structural systems can only be developed through complex iterative design process. Various best practices that lead to the development of reliable and robust spacecraft structures are reviewed.

NASA heritage programs such as Gemini, Mercury, Apollo, and the Space Shuttle are examined. Lessons learned from these programs are captured. To be able to build an appropriate structural system for a mission, design and mission requirements and the environment must be adequately defined. Then, trade studies and verification and validation need be performed. To build the structural system that performs as intended needs design, analysis, manufacturing and process control, testing, and quality assurance.

Since the first human space flight, the best practices for reliable and robust spacecraft structures appear to be well established, understood, and articulated by each generation of designers and engineers. However, the implementation of these best practices appears to be a problem. When the best practices are ignored or short cuts are taken, reliability suffers and risks accumulate. Program managers deviate from best practices due to the programmatic and resource (cost and schedule) issues brought on by anomalies and unpredicted problems, and unforeseen events. Thus for a reliable structural system, program managers need to be vigilant when anomalies and unforeseen problems arise that tend to violate best practices. 


\section{References}

1. Douglas, W. H., McIntosh, G. P., and Menegar, L. S., "Spacecraft reliability and qualification," Chapter 10, Gemini Mid-program Conference, NASA-SP 121, 1966, pp.89-99.

2. Dineen, R. C., "Development of the Gemini Launch Vehicle," Chapter 16, Gemini Midprogram Conference, NASA-SP 121, 1966, pp. 147-149.

3. Mitchell, W. B., Maynard, O. E., and Arabian, D. D., "Gemini results as related to the Apollo program," Chapter 22, Gemini Summary Conference, NASA-SP 138, 1967, pp. 329-336.

4. Smith, P. D., “Apollo experience report: Spacecraft structure subsystem,” NASA Technical Note TN D-7780, 1974.

5. Ryan, R. S., "A history of aerospace problems and their lessons," NASA Technical Paper TP-3653, 1996

6. Glynn, P. C. and Moser, T. L., "Orbiter structural design and verification," in Space Shuttle Technical Conference, N. Chaffee (Compiler), NASA Conference Publication 2342, Part 1, pp, 345-356, 1983.

7. Coldwater, H. G., Foll, R. R., Howell, G. J., and Dutton, O. J., " Space shuttle external tank performance improvements - The challenge," in Space Shuttle Technical Conference, N. Chaffee (Compiler), NASA Conference Publication 2342, Part 1, pp, 357-364, 1983.

8. Ryan, R. S., "Practices in adequate design," NASA Technical Paper TP-2893, 1989.

9. NASA NPR 7120.5C, "NASA Program and Project Management Processes and Requirements". 2006.

10. AIAA G-077-1998, Guide for the Verification and Validation of Computational Fluid Dynamics Simulations, 1998.

11. Verification \& Validation in Computational Solid Mechanics, ASME V\&V 102006, ASME, NY, NY.

12. DoD Instruction 5000.61, DoD Modeling and Simulation (M\&S) Verification, Validation, and Accreditation (VV\&A), 2006.

13. Ullman, D., "Less fudging on fudge factors," Machine Design, pp. 107-111, October 1986.

14. Haldar, A. and Mahadevan, S. Probability, Reliability and Statistical Methods in Engineering Design. Wiley and Sons, New York, 2000.

15. Nowak, A.S. amd Collins, K.R. Reliability of Structures. McGraw Hill, Boston, 2000 .

16. Wu, Y. -T., Burnside, O. H. and Cruse, T. A. "Probabilistic methods for structural response analysis," in W.K. Liu and T. Belytschko (Editors), Computational Mechanics of Probabilistic and Reliability Analysis. Elmpress, Switzerland, pp. 182-195, 1987.

17. Cruse, T. A., Burnside, O. H., Wu, Y. -T., Polch, E. Z. and Dias, J. B. "Probabilistic structural analysis methods for select space propulsion system structural components (PSAM)," Computers and Structures, Vol. 29 (5), pp. 891-901, 1988.

18. ANSYS General Purpose Finite Element Code, ANSYS Inc., Cannonsburg, PA, 2006. 
19. MSC-NASTRAN Finite Element Program, MSC Software Corporation, Santa Ana, CA, 2006.

20. NASA Safety Manual, NPG 8715.3, Jan. 24, 2000. 